\title{
Protée
}

\section{Accord réciproque. Pierce, Kandinsky et le potentiel dynamique du qualisigne}

David Scott

Volume 26, numéro 3, 1998

Logique de l'icône

URI : https://id.erudit.org/iderudit/030522ar

DOI : https://doi.org/10.7202/030522ar

Aller au sommaire du numéro

\section{Éditeur(s)}

Département des arts et lettres - Université du Québec à Chicoutimi

ISSN

0300-3523 (imprimé)

1708-2307 (numérique)

Découvrir la revue

Citer cet article

Scott, D. (1998). Accord réciproque. Pierce, Kandinsky et le potentiel

dynamique du qualisigne. Protée, 26(3), 17-23. https://doi.org/10.7202/030522ar
Résumé de l'article

Une des conceptions-clefs de la sémiotique peircienne est la structure relationnelle de la représentation - relation triadique entre representamen, objet, et interprétant et entre icône, indice et symbole. Un domaine privilégié pour l'étudier est fourni par l'art abstrait moderne : Wassily Kandinsky (18661944), par exemple, contemporain de Peirce (1839-1914) et sémioticien sans le savoir, comme artiste autant que comme théoricien de l'esthétique, a essayé de cerner les potentialités de la qualité pure. Cet article essayera de montrer comment la sémiotique peircienne, surtout dans le domaine de la théorie de l'iconicité, nous aide à mieux comprendre le potentiel dynamique des formes abstraites dans la peinture de Kandinsky, et de montrer comment sa théorie et sa pratique portent la marque des processus sémiotiques fondamentaux théorisés par Peirce. 


\section{ACCORD RÉCIPROQUE: \\ PEIRCE, KANDINSKY ET LE POTENTIEL DYNAMIQUE DU QUALISIGNE}
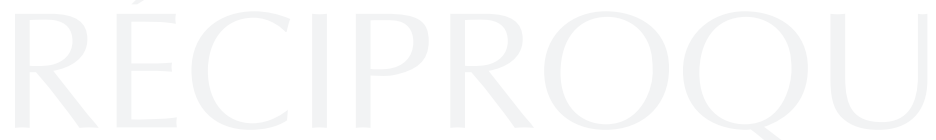

DAVID SCOTT

\section{INTRODUCTION}

Une des conceptions-clefs de la sémiotique peircienne est la structure essentiellement relationnelle de la représentation: d'un côté, la relation triadique entre representamen, objet, et interprétant; et de l'autre, la relation complexe, soit entre icône, indice et symbole à l'intérieur du même signe, soit entre des signes individuels iconiques, indiciaires ou symboliques mis en rapport les uns avec les autres. Un domaine privilégié - quoique relativement peu exploré - de l'étude de la qualité relationnelle de la sémiotique peircienne est celui de l'art moderne, et surtout de l'art géométrique abstrait. Car ce genre d'art est voué à l'expression des caractères qualitatifs de l'objet utilisant un «langage», en l'occurrence pictural, dont les propriétés sont principalement iconiques et dont les rapports, infiniment variables, se construisent autour de l'interaction des potentialités dynamiques des éléments mis en jeu. On se rappelle que l'un des grands contemporains de Peirce (1839-1914) est Wassily Kandinsky (1866-1944), le plus grand artiste moderne de l'art géométrique abstrait et, de plus, le théoricien le plus important des valeurs qualitatives de la représentation picturale. Sémioticien sans le savoir, Kandinsky, autant comme artiste que comme esthéticien, a essayé de cerner les potentialités de la qualité pure - formes et couleurs - afin de pouvoir les définir objectivement et, en tant qu'artiste, en créer de nouvelles combinaisons harmonieuses. Cet article va tenter d'établir un certain rapprochement entre les idées de Peirce et de Kandinsky afin de montrer comment la terminologie et les catégories sémiotiques, surtout dans le domaine de la théorie de l'iconicité, nous aident à mieux comprendre l'impact sur le récepteur de la dynamique des formes abstraites dans la peinture de Kandinsky; et, en même temps, de montrer comment la théorie et la pratique de l'art abstrait manifestent certains des processus sémiotiques fondamentaux théorisés par Peirce.

\section{UNE SÉMIOTIQUE DU DISCOURS PLASTIQUE NON FIGURATIF}

La question de ce qu'une sémiotique du discours plastique non figuratif pourrait nous apporter a été posée d'abord par Jean-Marie Floch, dans un article de 1981, qui choisit justement comme objet d'analyse un tableau de Kandinsky: Composition IV (1911) ${ }^{1}$. Pourtant, en l'occurrence, l'analyse de Floch, fondée sur la 
théorie sémiotique greimassienne, dont il observe scrupuleusement la terminologie (Floch, 1981: 136), insiste surtout sur les éléments iconographiques du tableau et en dégage le sens (potentiel) figuratif, en le mettant dans le contexte d'œuvres figuratives de Kandinsky datant de l'époque immédiatement précédente (1907-1910), dans lesquelles la portée religieuse ou autrement symbolique des signes picturaux est encore pleinement évidente. Ainsi, quoique Floch entreprenne une analyse formelle sémiotique assez rigoureuse de Composition IV, la divisant en trois segmentations et soulignant les oppositions (planaires, linéaires et chromatiques) à l'intérieur du tableau - au plan du signifiant - , l'expression de son contenu - au plan du signifié tend à être dégagée non pas uniquement à partir de ces éléments formels et de leurs rapports réciproques, mais d'un système de bricolage (ce terme est emprunté à Lévi-Strauss) d'éléments figuratifs hétéroclites (ibid.: 150-51) dont, d'après Floch, le tableau, en tant qu'énonciation, est structuré. Malgré donc la constatation par Floch de la substitution dans Composition IV d'une certaine "motivation poétique" à "l'immotivation des signes d'une sémiotique figurative" (ibid.: 155), son analyse retient celle-ci en l'occurrence comme alibi interprétatif. Quoique cet alibi soit sans doute encore possible et valable pour un tableau de cette étape dans l'évolution de l'œuvre de Kandinsky, il ne le sera plus à partir des années 20, quand débute la période des tableaux abstraits non figuratifs. Car le problème sémiotique posé par les peintures de l'époque Bauhaus de Kandinsky (19221933) et par celles de la période parisienne suivante (1933-1944) est précisément celui de la lecture d'un tableau apparemment sans référent ou «objet». À ce moment-là, l'énonciataire (pour utiliser la terminologie de Greimas), dépourvu d'alibis figuratifs, doit chercher d'autres points de repère sémiotiques. Et c'est ici que la sémiotique peircienne, avec sa conception plus élaborée que celle de la sémiologie greimassienne du fonctionnement des processus de l'interprétation - grâce surtout au concept de l'interprétant -, pourra nous aider à mieux cerner les problèmes de lecture posés par la peinture abstraite.
LE PROBlĖME SÉMIOTIQUE POSÉ PAR L'ART ABSTRAIT

Le problème sémiotique posé par l'art abstrait est surtout celui de son manque apparent d'objet. Car, s'il est sans objet, est-ce que le tableau abstrait est à proprement parler sémiotique, c'est-à-dire susceptible de fonctionner comme signe? Est-il susceptible d'une lecture sémiotique? Et si oui, quel est le statut sémiotique du signe «abstrait» et de son objet? Est-il autoréférentiel? ou symbolique d'une manière non spécifique? Le rôle de l'interprétant par rapport au signe «abstrait» est évidemment crucial ici. En principe, l'interprétant immédiat lit le signe par rapport à l'objet immédiat; or ici, l'objet immédiat n'est autre que le signe. Mais le tableau abstrait peut émettre en plus une certaine "aura» esthétique, fonction de la "poétisation" dont parle Floch (1981: 154). Quel est le rôle de l'interprétant dynamique dans l'interprétation de cette "aura» dans laquelle le representamen (qualisigne ou forme géométrique) baigne et qui indique peut-être un objet dynamique (conception englobante, harmonie supérieure) au-delà de l'objet immédiat? Et si cette "aura" sauve le signe abstrait de la tautologie inéluctable de l'autoréférence, comment se construit-elle?

S'il est évident que l'«aura» esthétique est fonction de l'interaction des lignes et des couleurs à l'intérieur du tableau, que peut-on dire de celles-ci? Que représente la forme abstraite en peinture? Un tableau est en principe une icône, mais une icône qui ressemble à quoi? Nous avons à notre disposition ici un choix de réponses tautologiques: le tableau ressemble à une icône; le tableau se ressemble; le tableau ressemble aux formes qu'il représente (points, lignes, couleurs). Toute icône présuppose un potentiel indiciaire. Est-ce que cet élément indiciaire nous sauve du cercle vicieux du même? En l'occurrence non, puisque la réponse à la question "Qu'est-ce qu'un tableau abstrait indique?» attire le même choix de tautologies: le tableau indique un indice; il s'indique; et il indique un certain ensemble de rapports ou de tensions internes. Enfin, toute icône a une fonction symbolique. Mais qu'est-ce qu'un tableau abstrait symbolise? Encore une fois, il symbolise un symbole; il se symbolise lui-même; mais quoiqu'il ne puisse, étant 
unique, remplir la fonction de signe conventionnel, c'est-à-dire un signe susceptible de répétition, il peut à la rigueur symboliser une certaine harmonie ou conception générale.

\section{THÉORIE DE L'ANALYSE SÉMIOTIQUE PEIRCIENNE} APPLIQUÉE À UN TABLEAU

L'analyse sémiotique du portrait de La Joconde de Léonard par Gérard Deledalle dans Théorie et pratique du signe (1979) ${ }^{2}$, quoique exemplaire en tant qu'exposé du fonctionnement des catégories peirciennes du signe, ne propose pas de solutions aux problématiques ponctuelles soulevées par l'art abstrait, surtout celle de la question de l'«aura» esthétique (dont le problème se pose d'ailleurs presque autant dans le contexte du tableau figuratif que dans celui de la peinture abstraite). Si, en effet, on soumet un tableau de Kandinsky, tel que Accord réciproque (1942), chefd'œuvre de la période parisienne, à une analyse analogue à celle que Deledalle propose pour $\mathrm{La}$ Joconde, on est frappé tant par les similarités que par les différences du processus de la sémiose relevées par la lecture des deux tableaux. Deledalle résume l'analyse du tableau de Léonard en tant qu'objet immédiat $(\mathrm{Oi})$ dans la formule suivante (ou $\mathrm{R}=$ Representamen; $\mathrm{O}=$ Objet; $\mathrm{I}=$ Interprétant $)$ :

$$
\mathrm{R}(1.2)=\mathrm{Oi}(2.1)\left\{\mathrm{Ii}_{(}\left(\mathrm{If}_{3}\right)(1.1,2.1,3.1), \operatorname{Id}_{1,2}, \mathrm{If}_{1,2}(1.2,2.2,3.1)\right\}
$$

- ce qui veut dire que le statut sémiotique du portrait de La Joconde est celui d'un sinsigne (1.2) constitué de qualisignes (1.1), perçu en tant qu'objet immédiat comme un signe iconique (2.1) interprété par l'interprétant immédiat et l'interprétant dynamique suivant des indices à l'intérieur du signe. Une lecture analogue d'Accord réciproque de Kandinsky produira sensiblement la même lecture, sauf que le titre et la signature de l'artiste étant donnés, le tableau de Kandinsky est un dicisigne (3.2) et non, comme c'est le cas avec le portrait, un rhème (3.1):

$$
\left.\mathrm{R}(1.2)=\mathrm{Oi}(2.1)\left\{\mathrm{Ii}_{(\mathrm{If}}\right)(1.1,2.1,3.1), \operatorname{Id}_{1,2}, \mathrm{If}_{1,2}(1.2,2.2,3.2)\right\}
$$

Deledalle exprime la relation de l'objet dynamique du signe avec ses interprétants dynamique et final comme suit:

$$
\mathrm{R}(1.2)=\operatorname{Od}(2.1)\left\{\operatorname{Id}_{2}, \mathrm{If}_{2}(1 \cdot 1,2.2,3.2), \mathrm{If}_{3}(1.2,2.3,3.2)\right\}
$$

L'expression du statut sémiotique de Accord réciproque de Kandinsky serait analogue, excepté que, mettant le tableau dans le contexte des écrits théoriques de Kandinsky, on pourrait peut-être classer celui-ci, du point de vue de l'interprétant, comme un argument, puisqu'il reflète les lois et conventions d'une esthétique rigoureuse systématiquement appliquée par l'artiste, ce qui nous donnerait:

$$
\mathrm{R}(1.2)=\operatorname{Od}(2.1)\left\{\operatorname{Id}_{2}, \mathrm{If}_{2}(1.1,2.2,3.2), \mathrm{If}_{3}(1.2,2.3,3.3)\right\}
$$

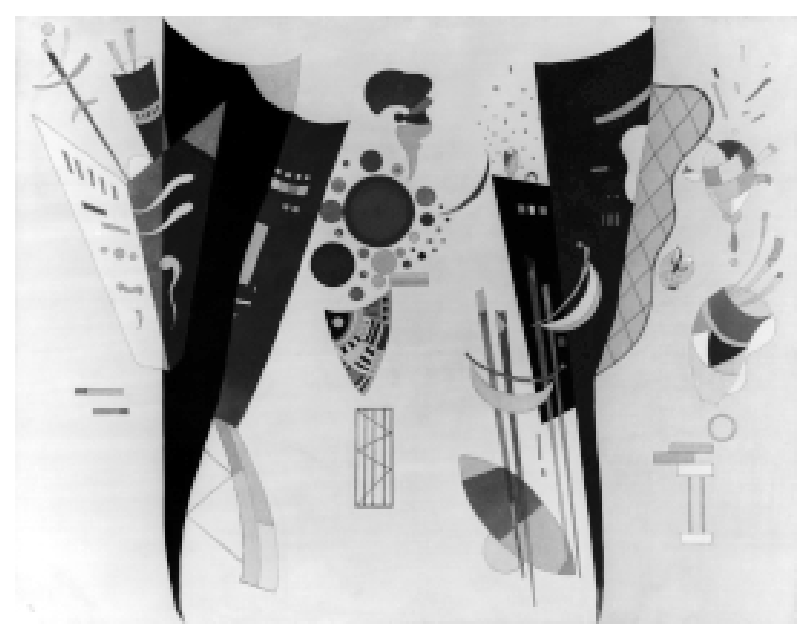

Kandinsky, Accord réciproque, 1942. Musée National d'Art Moderne (Paris).

\section{LA FORME COMME REPRESENTAMEN}

\section{ET LE TABLEAU COMME HYPOICÔNE}

C'est surtout la théorie de l'art abstrait et des qualités picturales pures élaborée par Kandinsky luimême dans ses essais magistraux De la spiritualité dans l'art (1914) et Point-ligne-plan (1926) qui nous permet de compléter et d'enrichir la sémiotique peircienne dans le domaine de l'iconicité, et surtout le concept de l'hypoicône, avec ses trois catégories d'image, de diagramme et de métaphore (C.P. 2.277) ${ }^{3}$. Prenons d'abord l'image qui, selon Peirce, consiste en des qualités - couleur, forme, ou autres qualisignes. Comment ces qualités travaillent-elles en tant que signes? La théorie esthétique de Kandinsky essaie justement de définir le potentiel sémiotique des 
formes ou qualités abstraites, ainsi que le potentiel dynamique de la couleur et de la forme. Passons ensuite au diagramme qui, selon Peirce, constitue une sorte d'analogie dyadique. Le tableau abstrait, en combinant des couleurs et des formes abstraites, crée un réseau diagrammatique; mais un diagramme de quoi? Quel est l'objet de ce diagramme? - est-ce un autre ensemble abstrait, un autre système de rapports, réel, mais non défini, sans étiquette ou référence, qui existerait derrière le signe? Là encore, la réflexion de Kandinsky sur sa pratique d'artiste proposera des réponses que nous approfondirons plus loin.

Finalement, qu'en est-il de la métaphore qui, suivant Peirce, représente le caractère représentatif d'un representamen en représentant un parallélisme dans quelque chose d'autre (C.P. 2. 277)? Si la combinaison de couleurs et de formes qui constitue le tableau abstrait est envisagée comme une unité ou une totalité, celle-ci peut à son tour être envisagée comme métaphorique. Pourtant, puisque seul est donné l'un des deux termes de la métaphore (l'image et non le référent), le référent reste à dégager. D'ailleurs, du point de vue de la métaphore, l'intérêt du tableau abstrait est peut-être moins le problème de l'interprétation (Kandinsky évite des titres «littéraires» ou "parlants») que la manière dont un potentiel abstrait métaphorique se construit sans indiquer un objet spécifique et sans se figer dans un symbole qui appellerait une lecture définitive.

La façon dont Kandinsky évite des titres ${ }^{4}$, qui proposeraient «une clef» permettant d'ouvrir ses tableaux à une signification métaphorique précise, est révélatrice de leur statut sémiotique: car le tableau ou "Composition" de Kandinsky est une représentation (métaphorique) d'une composition. Ce statut, placé en quelque sorte entre parenthèses, de la dimension métaphorique du signe que constitue le tableau souligne, comme nous venons de le voir, la structure tautologique du signe esthétique abstrait. Comme Peirce l'a bien compris, dans l'icône, il n'y a parfois pas d'objet ou, plus exactement, le rôle de l'objet est joué par l'interprétant (C.P. 2.311). Le sentiment esthétique, placé par Peirce dans la catégorie de Priméité (C.P. 5.111), est fonction dans les tableaux de
Kandinsky d'une perception plus riche ou approfondie de ce qu'est une composition, de ce qu'est un signe: l'interprétant saisit à travers le representamen «abstrait» non pas un objet, mais les qualités qui seraient susceptibles d'établir un pur rapport et que l'interprétant dynamique doit induire en tirant sur ses expériences collatérales (son expérience préalable, par exemple, de la lecture des signes esthétiques et du plaisir qui y est parfois associé). Les «relations pures» dont parle Kandinsky dans le contexte de la structure autoréflexive de la composition sont saisies, selon Peirce par la conscience qui devient consciente de ses propres procédés et où le sentiment esthétique arrive à se représenter à lui-même comme représentation (C.P. 5.71).

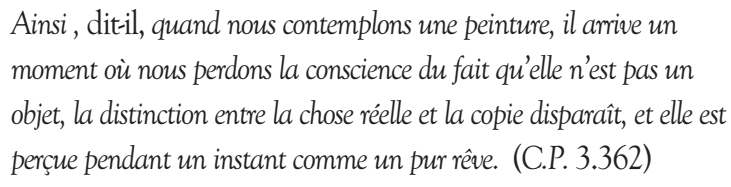

Cette perception de l'image visuelle comme pure possibilité et de la conscience de l'autoréflexivité de la vision se retrouve dans le livre de Michel Henry sur la peinture abstraite de Kandinsky, intitulé Voir l'invisible, dans lequel il est affirmé que la peinture abstraite «fait voir en tant qu'elle rend la vision à elle-même, accroît sa capacité de voir, dans l'intensification de son pathos et par lui " ${ }^{5}$. La thèse de Henry est que la motivation des signes picturaux vient de l'intérieur, répondant à une nécessité qui exprime une appréhension subjective ou «pathétique» de la Vie. Le visible du tableau est donc inspiré par un contenu «invisible»:

Quand il sera établi que la composition d'un tableau repose sur l'élément intérieur et se construit à partir de lui, l'évidence de cette vérité cruciale se tiendra devant nous: que la peinture, qui est cette composition, n'est pas tributaire du visible. Elle ne trouve en lui ni ses lois ni son principe, puisqu'elle dépend de ce qui s'est dédoublé et exprime ce dédoublement.

(Henry, 1988: 85)

Quoique Henry comme Peirce écarte de son propos la psychologie et les théories de la mimesis, et poursuive son argument d'une manière rigoureusement logique, son manque de terminologie et d'instruments 
d'analyse sémiotique (voir, par exemple, ibid.: 152-3)

fait que sa thèse tend à tourner court, tournant même finalement en rond dans un tourbillon

phénoménologique, où des concepts généraux comme «la Vie» ou «la Nécessité intérieure» sont à maintes reprises affirmés sans pour autant approfondir notre compréhension de leurs opérations.

Une analyse aussi fine mais beaucoup plus riche en aperçus du problème de la nature de l'art abstrait, du point de vue du récepteur autant que de l'artiste, est offerte par Dee Reynolds dans son livre admirable, Symbolist Aesthetics and Early Abstract (1995). Dans cette étude comparative de la poétique de Mallarmé et Rimbaud et de l'esthétique de Kandinsky et Mondrian, en partie inspirée par la sémiotique peircienne, Reynolds souligne le rôle vital joué par l'imagination du spectateur dans la lecture de l'espace «imaginaire" proposé par le tableau abstrait. Non seulement cette imagination tire-t-elle de multiples et diverses ressources de la mémoire et de l'expérience collatérale, mais elle sait aussi répondre à l'aspect dynamique de l'objet en y investissant des énergies correspondantes. Par exemple, selon Reynolds, les connotations figuratives imputées aux formes abstraites des peintures de Kandinsky

[...] sont imaginées par le spectateur, et leurs ressemblances aux organismes vivants introduisent une dimension «métaphorique» qui, elle-même, tire l'attention du spectateur vers la capacité de l'imagination d' "animer» les éléments picturaux. D'ailleurs, la fonction des «signes" picturaux est de ressembler aux signes, mais sans renvoyer pour autant à aucun système codé: c'est ainsi $q u$ 'ils arrivent à jouer avec l'attente du spectateur en signalant leur propre illisibilité en tant que signes. (Ibid.: 151-2)

À la différence, donc, du plaisir esthétique fourni par la peinture figurative, où entre un certain plaisir de reconnaissance du monde visible, celui fourni par le tableau abstrait n'est pas une fonction de son potentiel référentiel mais de son potentiel sémiotique. Ce qui importe, ce n'est pas tant le visible en soi, que la façon dont le visible peut se reconstruire selon des exigences intérieures, qui jouent un rôle indispensable dans le jeu des signes. Ce potentiel est relativement peu motivé. Le récepteur jouit des qualisignes en tant que potentialités sémiotiques - la couleur pour la couleur, la forme pour la forme -, l'«aura" esthétique de ces couleurs et de ces formes étant en partie fonction des potentialités dynamiques qu'elles recèlent. Dans ses traités théoriques sur la représentation abstraite dans l'art, Kandinsky montre que même la plus infime des marques graphiques point, ligne ou plan - a un potentiel dynamique et donc sémiotique, et que la page blanche - papier ou toile - qui constitue le support du signe pictural est déjà chargée d'un potentiel sémiotique. De même pour la couleur dont Kandinsky analyse la dynamique centrifuge ou centripète, les valeurs "chaudes" et "froides». En ce qui concerne les rapports entre ces éléments - formels ou chromatiques -, il est évident qu'une composition de Kandinsky ne signifie strictement rien, sa fonction étant plutôt de montrer la complexité dynamique de l'interaction des diverses énergies et qualités qui la constituent - ou, comme dirait Peirce, «la Forme d'une Relation» (C.P. 4.530).

En termes peirciens, on pourrait dire qu'un tableau de Kandinsky aspire surtout au statut sémiotique du diagramme, une forme de l'hypoicône, où les icônes qui la constituent entrent dans des rapports indiciaires les unes avec les autres. De plus, pour Peirce, un diagramme est une hypoicône dans laquelle non seulement l'objet du representamen peut devenir en même temps son interprétant, mais aussi dans laquelle l'objet n'est pas une idée ou une chose mais «la forme d'une relation", précisément la relation qui lie les parties correspondantes d'un diagramme (C.P. 4.530). Le tableau abstrait se construit donc comme un signe pur, dans lequel les opérations interprétatives ne visent plus un sens mais cherchent plutôt la possibilité de significations indéfiniment différées. Selon l'hypothèse de Peirce, «l'état d'esprit devient le plus purement esthétique quand il reste parfaitement naif, sans jugement critique, et que le critique esthétique fonde ses jugements sur l'expérience d'un tel état pur et naif»" (C.P. 5.111). C'est ainsi qu'une composition de Kandinsky nous offre la possibilité de faire l'expérience des qualités pures, de reconnaître des signes tout en faisant abstraction - littéralement - de leurs objets. 


\section{SÉMIOTIQUE DE LA COULEUR ET DE LA FORME CHEZ KANDINSKY}

Tout en regrettant que "Le spectateur [soit] également trop habitué à chercher [...] "un sens" dans un tableau" (Kandinsky, 1914: 49), Kandinsky souligne, dans son essai Du spirituel dans l'art, le fait que l'art abstrait appelle un nouveau genre de lecture: le récepteur ne cherche plus le sens du tableau ni le rapport entre son contenu et quelque objet du monde "réel», mais plutôt une compréhension de la logique ou de la dynamique des rapports entre les formes et les couleurs à l'intérieur du cadre. Cette lecture ouvre sur un monde "spirituel", dans le sens où le tableau cherche une réponse dans l'esprit du récepteur qui appréhendera et jouira des rapports à l'intérieur de l'image sans référence au monde extérieur. En d'autres termes, le signe constitué par le tableau renvoie à l'interprétant à la fois comme objet et comme dispositif interprétatif: le circuit triadique de la production du sens (la sémiose) est court-circuité et deux des trois éléments du processus (à savoir l'objet et l'interprétant) sont intériorisés; le signe seul reste à l'extérieur. «L'espace tridimensionnel» (ibid.: 172) que la toile abstraite devient, avec sa superposition de plans, est reflété par l'esprit qui devient, lui aussi, un espace imaginaire (cf. Reynolds, 1995) dans lequel les rapports entre les éléments qui constituent le signe sont pesés en un double échange dialectique: celui de interprétant/objet à l'intérieur de l'esprit et celui extérieur/intérieur du rapport signe/interprétant. « $L a$ nécessité intérieure» du tableau abstrait (Kandinsky, 1914: 169) est reflétée par le besoin intérieur de l'esprit en un échange stable mais infiniment variable.

Mais quels sont les éléments qui constituent la composition abstraite et quelles sont la logique et la dynamique qui les structurent? Kandinsky dit que la composition

[...] est une construction de formes colorées et dessinées, qui existent indépendamment en tant que telles, procédant de la nécessité intérieure et formant, par cette voie commune ainsi crée, un tout que l'on nomme tableau. (Ibid.: 169)

En ce qui concerne la couleur d'abord, il affirme que sa puissance expressive est beaucoup plus riche si elle n'est pas associée directement à des objets existant dans le monde réel; c'est en tant que valeur pure que la couleur arrive le mieux à exprimer un «sens intérieur». Dans son chapitre «Le Langage des formes et des couleurs" (ibid.: 113-72), il élabore tout un système de valeurs relatives, qui comprend et dépasse la loi de la complémentarité des couleurs établie par Chevreul au 19 e siècle. Pour simplifier, il suffira de noter ici que les trois couleurs primaires (jaune, rouge, bleu) et les trois couleurs secondaires (vert, orange, violet), en tant que qualisignes, recèlent une dynamique latente. Là où le rouge contient son propre mouvement, les couleurs "chaudes", le jaune et l'orangé, l'expulsent suivant une dynamique excentrique ou centripète. Les couleurs «froides» par contre, le bleu et le violet, ne rayonnent pas mais absorbent le mouvement suivant une dynamique concentrique. Le vert, mélange de l'élément dynamique qu'est le jaune et de la profondeur du bleu, reste le plus calme et immobile des tons.

Un sous-titre projeté de l'essai Point-ligne-plan de Kandinsky (1926), "Pour une grammaire des formes", souligne le projet sémiotique du peintre, presque aussi ambitieux que celui de Peirce, qui essaie de classer toutes les formes possibles du signe. Tout comme chez Peirce, le projet sémiotique n'est pas celui, impossible, de classer l'infini des phénomènes mais plutôt de répartir les signes en des catégories restreintes. Ainsi, pour Kandinsky, une grammaire des formes établirait non pas l'inventaire de tous les objets que l'on pourrait représenter, mais de toutes les qualités qui entrent en jeu dès qu'on fait des marques graphiques sur un support quelconque. Comme Peirce, Kandinsky choisit un système triadique de classement - point, ligne, plan - qui correspond d'une manière générale à la triade symbole, indice, icône. Le plan, comme l'icône, constitue à la fois le support et la forme essentielle de l'image visuelle; la ligne est l'élément actif qui, à l'intérieur du plan/icône, indique, relie et trace, créant ainsi une sorte de réseau diagrammatique; finalement, le point est, comme le symbole peircien, l'élément primordial qui sous-tend toutes les autres fonctions sémiotiques; comme Kandinsky le dira, le point est le degré zéro qui 
représente «l'ultime et unique union $d u$ silence et de la parole" (Kandinsky, 1926: 33). Comme le symbole, le point peut rester invisible, occulté par les initiatives plus démonstratives de la ligne/indice ou du plan/ icône, ou peut s'affirmer lui-même en tant qu'indice ou icône. Les diagrammes qui accompagnent le texte de Kandinsky montrent d'une manière systématique les métamorphoses du point qui, en se liant, se transforme en ligne et, en s'agrandissant, en plan; ces transformations constituent en outre une démonstration graphique des transformations que le symbole est susceptible de subir.

Quant à la ligne, fût-elle géométrique ou improvisée, les diverses dynamiques de ses multiples périples sont notées par Kandinsky, qui n'oublie jamais de nous rappeler les implications pour le plan du mouvement linéaire: chaque ligne, d'une manière ou d'une autre (en circonscrivant, en structurant, ou même en oblitérant), indique le plan, tout comme l'indice indique, utilise ou parfois devient une icône. Finalement, le plan lui-même est envisagé d'abord comme un champ abstrait de potentialités qui seront activées par l'empreinte du point et de la ligne. Comme l'icône, le plan n'est pas obligé de représenter une forme complète, mais peut exprimer un sentiment ou une tension avec la collaboration minimale d'une ligne ou d'un point. Ainsi, par exemple, les diagonales, auxquelles Kandinsky attachait tant de prix, sont des icônes qui, selon leur orientation, expriment une harmonie ou une discordance, tandis que les verticales expriment un "calme chaud» et les horizontales un "calme froid». Un simple cercle, en tant qu'icône, devient «léger» s'il flotte en haut de la page, mais devient lourd comme une pierre s'il descend vers le bas. Toutes ces marques, comme les signes peirciens, ne sont que des potentialités dont la signification ultime ne se manifeste que quand elles réagissent réciproquement dans des configurations concrètes, ce dont les tableaux de Kandinsky constituent des réalisations exemplaires.

La capacité qu'ont les signes abstraits à créer des sentiments esthétiques ou des harmonies complexes est surtout enrichie par l'interaction de qualités provenant de différents domaines, dont les deux principaux ouverts à la peinture sont la forme et la couleur. C'est par l'interaction des différents plans, facilitée surtout par les différentes qualités de rayonnement ou d'absorption de la couleur mises en rapport avec la dynamique spatiale des lignes et le poids variable des points, que la composition abstraite arrive à créer cet «espace tridimensionnel» (Kandinsky, 1914: 172), qui devient le miroir de l'espace intérieur de l'esprit du récepteur. C'est cette primauté, dans la peinture de Kandinsky, des qualités pures organisées dans des relations pures qui nous permet de mieux comprendre peut-être tant les processus de l'esprit que le fonctionnement des signes. Ses tableaux sont des diagrammes, esthétiques et scientifiques, reflétant et flattant ce besoin que nous éprouvons de faire des rapports, qui semble fonder tout à la fois l'esprit humain et la sémiotique.

\section{NOTES}

1. J.-M. Floch, $1981: 135-57$.

2. G. Deledalle, 1979: 117-29.

3. Selon la convention, les extraits des Collected Papers (C.P.) sont référencés par volume et paragraphe.

4. Dans la période "parisienne" (1933-44) surtout, Kandinsky propose des titres à ses œuvres, tels que Accord réciproque, Courbe dominante, Action variée, Succession, etc., qui suggèrent un certain potentiel métaphorique, mais dont la fonction première cependant est de proposer une lecture strictement formelle du tableau.

5. M. Henry, 1988: 51.

\section{RÉFÉRENCES BIBLIOGRAPHIQUES}

FLOCH, J.-M. [1981]: «Sémiotique d'un discours plastique non figuratif ", Communications, 24, 135-57.

DeLEDALLE, G. [1979]: Théorie et pratique du signe. Introduction à la sémiotique de Charles S. Peirce, Paris, Payot.

Henry, M. [1988]: Voir l'invisible. Sur Kandinsky. Essai, Paris, Éd.

François Bourin.

KANDINSKY, W. [1914]: Du spirituel dans l'art, et dans la peinture en particulier (dir. Philippe Sers), Paris, Denoël, coll. « Folio Essais, 1989;

[1926]: Point-ligne-plan. Contribution à l'analyse des éléments picturaux (sous la dir. de P. Sers), Paris, Denoël-Gonthier, 1970.

PeIRCE, C. S. [1931-35]: Collected Papers, vol. I-V (sous la dir. de

C. Hartshorne et P. Weiss), Cambridge, Mass., The Belknap Press;

[1958]: Collected Papers, vol. VII-VIII (sous la dir. de A. W. Burks), Cambridge, Mass., The Belknap Press (trad. D. Scott).

ReYNOLDS, D. [1995]: Symbolist Aesthetics and Early Abstract Art. Sites of Imaginary Space, Cambridge, Cambridge University Press (trad. D. Scott). 